\title{
Fascial sling technique for dural reconstruction after translabyrinthine resection of acoustic neuroma: technical note
}

\author{
James K. Liu, M.D., ${ }^{1-3}$ Smruti K. Patel, B.A., ${ }^{1}$ Amanda J. Podolski, B.A., ${ }^{1}$ \\ AND ROBERT W. JYung, M.D.,3 \\ Departments of ${ }^{1}$ Neurological Surgery and ${ }^{2}$ Otolaryngology-Head and Neck Surgery, and ${ }^{3}$ Center for Skull \\ Base and Pituitary Surgery, Neurological Institute of New Jersey, University of Medicine and Dentistry of \\ New Jersey, New Jersey Medical School, Newark, New Jersey
}

\begin{abstract}
Reconstruction of presigmoid dural defects after resection of acoustic neuromas via the translabyrinthine approach is paramount to prevent postoperative CSF leakage. However, primary dural reapproximation and achieving a watertight closure of the dural defect in this anatomical region are quite difficult. Standard closure techniques after the translabyrinthine approach often involve packing an abdominal fat graft that plugs the dural defect and mastoidectomy cavity. This technique, however, may pose the risk of direct compression of the fat graft on the facial nerve and brainstem. Nonetheless, even with the evolution in dural repair techniques, postoperative CSF leaks can still occur and provide a route for infection and meningitis. In this report, the authors describe a novel dural "sling" reconstruction technique using autologous fascia lata to repair presigmoid dural defects created after translabyrinthine resection of acoustic neuromas. The fascia lata is sewn to the edges of the presigmoid dural defect to create a sling to suspend the fat graft within the mastoidectomy defect. A titanium mesh plate embedded in porous polyethylene is secured over the mastoidectomy defect to apply pressure to the fat graft. In the authors' experience, this has been a successful technique for dural reconstruction after translabyrinthine removal of acoustic neuromas to prevent postoperative CSF leakage. There were no cases of CSF leakage in the first 8 patients treated using this technique. The operative details and preliminary results of this technique are presented. (http://thejns.org/doi/abs/10.3171/2012.6.FOCUS12168)
\end{abstract}

KEY WoRDS • dural sling reconstruction $\bullet$ autologous fascia $\bullet$
cerebrospinal fluid leak $\bullet$ translabyrinthine approach $\bullet$ petrosectomy
acoustic neuroma $\bullet$ skull base surgery

$\mathrm{T}$ He translabyrinthine approach to the cerebellopontine angle is a versatile cranial base approach for the removal of both small and large acoustic neuromas. ${ }^{14,16,22}$ This approach is generally preferred for removal of all acoustic tumors, resulting in nonserviceable hearing, and also for large tumors $(>3 \mathrm{~cm})$ when the likelihood of hearing preservation is low. ${ }^{4}$ By using the presigmoid corridor, this approach offers the advantage of virtually no cerebellar retraction as well as early identification of the facial nerve at the distal IAC. First described in 1904 by Panse, ${ }^{21}$ the translabyrinthine approach was not fully developed and standardized until 1964 by House and Hitselberger. ${ }^{13}$ Translabyrinthine resection of acoustic neuromas requires a presigmoid dural

\footnotetext{
Abbreviation used in this paper: IAC = internal auditory canal.
}

opening that extends over the IAC and results in a skull base dural defect, which has historically been difficult to reconstruct. ${ }^{23}$ These surgeries are often complicated by postoperative CSF leaks and fistula formation, which increases the incidence of development of other complications such as wound infection and meningitis. 5,27,30 Postoperative CSF leak rates for all acoustic neuroma surgery have been reported to range between $0 \%$ and $30 \%$ in the literature, but the mean leak rate remains approximately 10\%.,8-10,15,17,26,27 The postoperative CSF leak rates after translabyrinthine removal of acoustic neuromas range from $0 \%$ to $13 \%$ as reported in the most recent literature. $3,6,7,11,18,19,29$ Although appropriate medical and surgical management of CSF leaks is generally feasible, the ultimate goal is directed at prevention of postoperative CSF leakage. Achieving a watertight dural closure of 
these presigmoid dural defects after translabyrinthine resection poses a significant challenge to the acoustic neuroma surgeon. A meticulous reconstruction and dural closure technique is required for the prevention of CSF leak formation.

Traditionally, the standard closure technique when using the translabyrinthine approach involves an abdominal fat graft that is packed through the dural defect and mastoidectomy cavity. ${ }^{10,31}$ In this technique, the fat graft is in direct contact with the facial nerve, brainstem, and cerebellum. When the fat is overpacked and under pressure, symptomatic compression of the brainstem, cerebellum, and facial nerve can occur. ${ }^{15}$ Other complications of autologous fat grafting include fat necrosis, subarachnoid fat embolism, lipoid meningitis, wound discharge, and local fistula formation. ${ }^{23-25,28}$ Various other dural reconstruction techniques have been described, including covering the defect with a free temporalis muscle graft or a pedicled temporal fascial flap. ${ }^{20}$ Some have also described occlusion of mastoid and temporal bone air cells using bone wax, bone paste, fat, muscle, and hydroxyapatite cement and titanium mesh., ${ }^{1,2}$ However, even with the evolution of current dural repair techniques for reconstruction after translabyrinthine surgery, postoperative CSF leak rates are still reported to be as high as $9.5 \%{ }^{27}$

In this report, we describe a novel dural "sling" reconstruction technique to repair presigmoid dural defects created after a translabyrinthine approach for removal of acoustic neuromas. An autologous fascial graft is sutured to the presigmoid dural defect to suspend the fat graft within the mastoidectomy defect. This technique prevents the fat from coming in direct contact with the facial nerve and brainstem. In addition, less fat is required to occlude the mastoid cavity. The operative technique is described and illustrated. We also report the results of our preliminary experience using this technique in a small cohort of patients.

\section{Operative Technique}

A standard translabyrinthine approach is performed to remove the acoustic neuroma. The presigmoid dura is incised in a T-shaped fashion that extends directly over the IAC (Figs. 1A and 2A). After removal of the tumor, the cerebellopontine angle structures including the facial nerve, brainstem, and cerebellum are visualized within the dural defect (Figs. 1B and 2B). Due to retraction and shrinkage of the dural edges, primary dural approximation is not possible. A dural sling is created by suturing a piece of autologous fascial graft (either abdominal fascia or fascia lata from the thigh) to the edges of the dural defect using interrupted 4-0 Nurolon (Ethicon) sutures (Figs. 1C and 2C). A redundant portion of the fascial graft is carefully placed over the contents of the IAC as an onlay because this area cannot be directly sutured. A monolayer of Surgicel (Ethicon) is then placed over the suture line. The aditus ad antrum is occluded with a small muscle graft followed by hydroxyapatite cement (HydroSet, Stryker) to prevent any fistulous communication into the middle ear. Any visible mastoid air cells are occluded with bone wax or cement. Once the fascial sling is se-
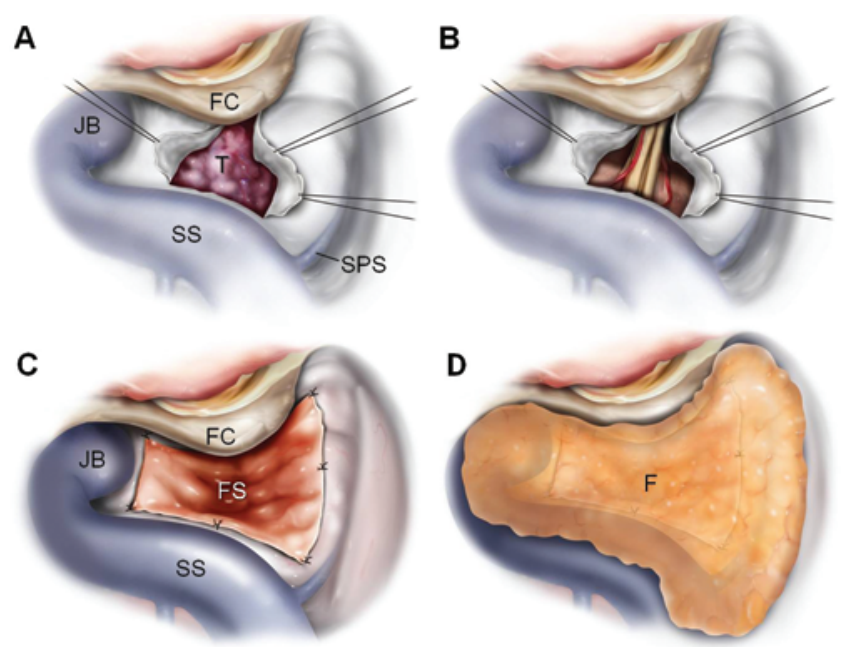

Fig. 1. Illustrations demonstrating the fascial sling technique for translabyrinthine reconstruction using a left-sided approach. A: The tumor $(T)$ is exposed after a T-shaped presigmoid dural incision. B: The facial nerve is preserved and visualized after tumor removal. $\quad$ C: An autologous fascial sling (FS) is sutured into the edges of the presigmoid dural defect using interrupted 4-0 Nurolon sutures. A redundant portion of the fascial graft is carefully placed over the contents of the IAC as an onlay, because this area cannot be directly sutured. D: Once the fascial sling is secured in place, an autologous fat graft $(F)$ is placed on top of the dural sling to fill the mastoid cavity. FC = fallopian canal; JB = jugular bulb; SPS = superior petrosal sinus; SS = sigmoid sinus. Reproduced with permission. Copyright Neurological Institute of New Jersey.

cured in place, an initial autologous fat graft is placed on top of the dural sling within the deeper aspect of the mastoid cavity (deep to the level of the fallopian canal; Fig. 2D). This initial fat layer is used to occlude any areas of CSF egress, and care is taken not to overpack the defect so as to avoid compression of the facial nerve. Continuous facial nerve monitoring is used during the reconstruction to detect any nerve irritation. Fibrin glue is placed over this initial fat graft followed by another layer of Surgicel (Fig. 2E).

The remaining superficial mastoid cavity is filled with another layer of autologous fat graft (Figs. 1D and 2F). Care is taken to avoid compression of the sigmoid sinus, as this can sometimes result in venous outflow obstruction and possible venous hypertension. ${ }^{15}$ The fat graft is then bolstered by a titanium mesh plate embedded in porous polyethylene (Medpor Titan implant, Stryker). The plate is secured to the edges of the bony mastoid defect with titanium screws. Meticulous multilayered wound closure is then performed. We do not use routine postoperative lumbar drainage after translabyrinthine approaches to avoid complications of intracranial hypotension.

\section{Results}

Eight patients ( 5 male and 3 female, mean age 55.6 years) underwent translabyrinthine removal of acoustic neuroma followed by subsequent autologous fascial dural sling reconstruction. Fifty percent of the tumors were left-sided and the remaining 50\% were right-sided. One patient also had concomitant neurofibromatosis Type II. 

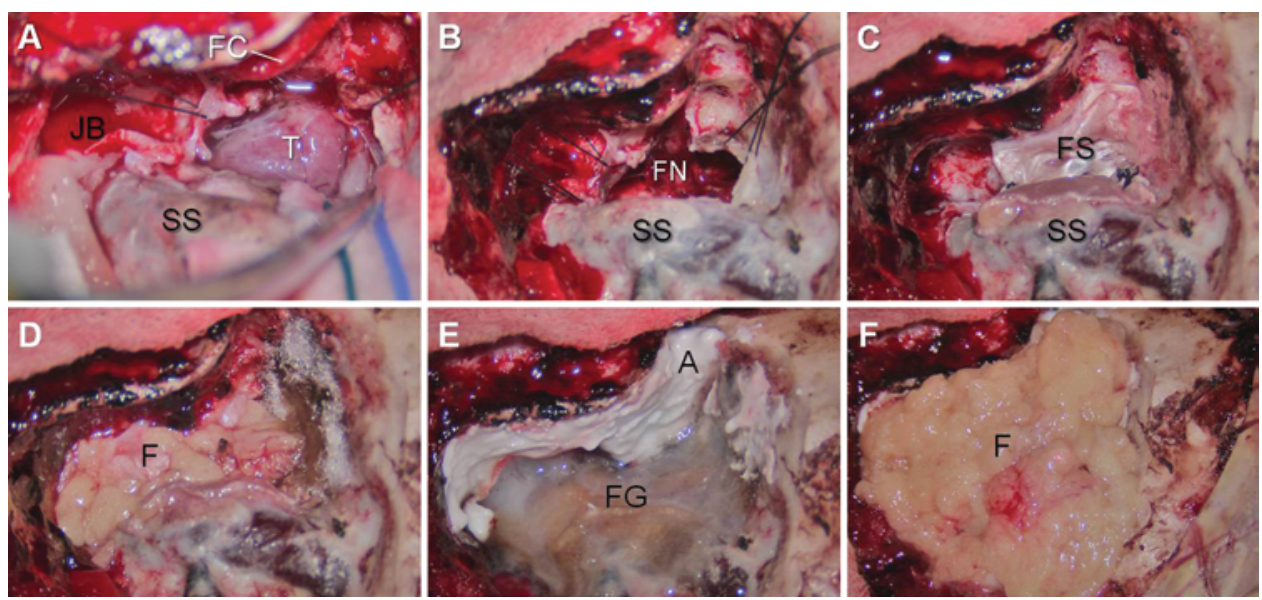

FIG. 2. Intraoperative photographs demonstrating fascial sling reconstruction after translabyrinthine resection of a left-sided acoustic neuroma. A: Tumor $(T)$ is exposed through a left-sided presigmoid dural incision. B: After tumor removal, facial nerve (FN) is identified and preserved in the presigmoid dural defect. C: An autologous fascial sling (FS) is sewn into the edges of the presigmoid dural defect using interrupted 4-0 Nurolon sutures. The redundant portion of the fascial graft is carefully placed over the facial nerve of the exposed IAC as an onlay graft because this area cannot be directly sutured. D: An initial deep fat graft (F) is placed over the fascial sling to occlude any areas of CSF egress. E: The aditus ad antrum (A) and remaining mastoid air cells are occluded with hydroxyapatite cement. A monolayer of Surgicel and fibrin glue (FG) is placed over the initial deep fat layer. $\quad F$ : The remaining superficial mastoid cavity is filled with another layer of autologous fat graft $(F)$.

In $50 \%$ of the patients, abdominal fascia was used as the dural sling, and in the remaining cases, fascia lata was used. The mean follow-up period was 13.8 months (range 6-22 months).

Patients were evaluated for the presence of postoperative CSF leakage (incisional leak, rhinorrhea, and otorrhea). There were no postoperative CSF leaks (0\%) after fascial sling reconstruction. We compared these results to our own historical control group $(n=5,1$ male and 4 females, mean age 49.6 years) in which a dural substitute (DuraGen [Integra LifeSciences] or Dura Matrix [Stryker]) was used as the sling material, instead of autologous tissue. Mean follow-up in these patients was 35.8 months (range 30-45 months). In this control group, 1 (20\%) of the 5 patients presented with a postoperative CSF leak (incisional leak), which was subsequently repaired and required lumbar drain placement.

\section{Discussion}

Cerebrospinal fluid leakage remains a common complication following removal of acoustic neuromas via the translabyrinthine approach. The presigmoid dural defect created during the surgery often cannot be primarily reapproximated in a watertight fashion. Exposure of the temporal bone air cells and aditus ad antrum provides multiple potential pathways for CSF fistula formation, thus providing a route for resultant infection and meningitis. Because of the morbidity and mortality associated with these postoperative complications, an effort must be made to prevent CSF leakage. The presentation of CSF leaks may range from incisional, otorrhea (CSF leakage through the tympanic membrane), and rhinorrhea (CSF leakage through the eustachian tube and nasopharynx). ${ }^{12,30}$ Following the transmastoid transtemporal petrosectomy required for the translabyrinthine approach, the mastoid antrum and mastoid air cells become exposed, enabling CSF to leak through if the air cells are not properly sealed..$^{30}$ Dural opening into the posterior cranial fossa provides direct communication between the subarachnoid space and the mastoid cells. The CSF can then enter the middle ear via the aditus ad antrum, sinus tympani cells, facial recess cells, or retrofacial air cells. ${ }^{12}$ Cerebrospinal fluid can also reach the temporal bone through apical air cells, which are located above and below the IAC. Therefore, it is critical to obliterate the mastoid antrum and remaining mastoid air cells, in addition to achieving a watertight dural closure to prevent fistulous pathways for CSF leakage. Meticulous multilayer soft-tissue closure of the wound followed by placement of a compressive mastoid pressure dressing is also important for preventing pseudomeningocele formation and incisional CSF leakage.

One common technique that has been used to reduce the occurrence of CSF fistula formation and leakage is packing or plugging the dural defect and mastoid cavity with an autologous fat graft. However, fat packing may pose a potential danger if overpacking results in mass effect on the brainstem, cerebellum, and/or cranial nerves. In addition, although rare, autologous fat grafting may result in complications including lipoid meningitis, subarachnoid fat embolism, fat liquefaction, and fat necrosis. ${ }^{23-25,28}$ In this report, we describe a novel reconstructive technique in which a dural sling is created at the base of the mastoid defect, in which the fat graft is suspended (Fig. 3). The sling serves several purposes. First, the fascial graft provides coverage of the presigmoid dural defect, thereby converting a large dural defect with a "highflow" CSF leak state to a "low-flow" or minimal CSF leak state. Second, the fascial sling allows suspension of the fat graft to avoid overpacking of fat, which can run the risk of direct compression on the facial nerve or brainstem. The sling also minimizes the volume of fat packing needed to prevent CSF egress through the dural repair. 
J. K. Liu et al.
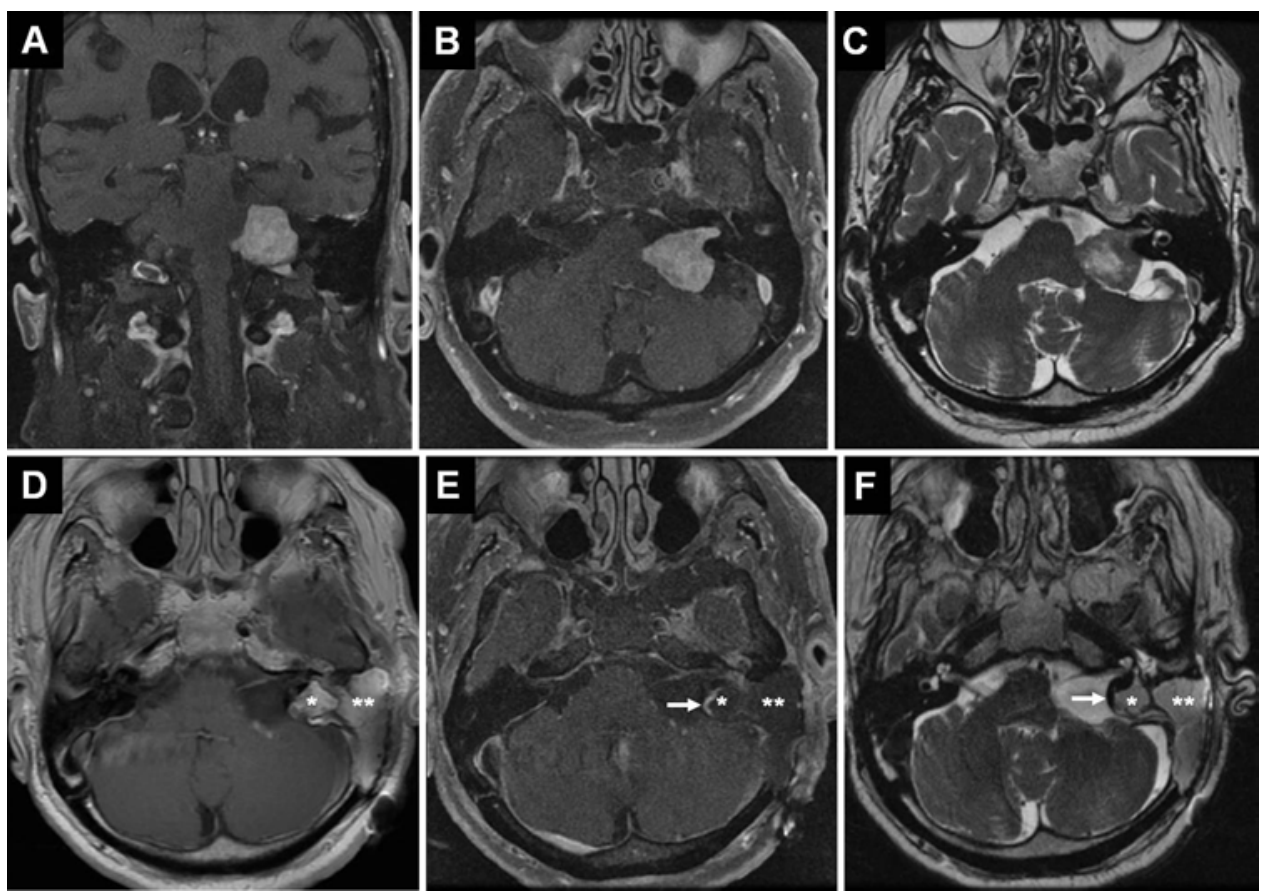

FIG. 3. Preoperative $(A-C)$ and postoperative (D-F) MR images. A-C: Coronal $(A)$ and axial $(B) T 1$-weighted images after $\mathrm{Gd}$ administration and an axial T2-weighted image (C) demonstrate a left-sided acoustic neuroma within the cerebellopontine angle with tumor extension into the fundus of the IAC. A left-sided translabyrinthine approach was performed followed by fascial sling reconstruction. D-F: Axial T1-weighted images with Gd administration (D) and fat suppression (E), and a T2-weighted image $(F)$ demonstrate complete tumor removal with excellent decompression of brainstem. The fascial sling (arrow) allows suspension of the fat graft within the mastoid cavity. Note that the fat graft is not in any direct contact with or compression against the facial nerve, brainstem, and cerebellum. This technique also minimizes the volume of fat required for reconstruction. ${ }^{*}$ deep fat layer; ** superficial fat layer.

Although synthetic graft materials (dural substitute) can be used for the sling, we believe that autologous fascial tissue is a better source for dural reconstruction and postoperative healing than dural allografts. In our report, the autologous fascia group had a lower rate of CSF leak than the dural substitute group ( $0 \%$ vs $20 \%$, respectively). The senior author has had experience with 2 prior cases (unpublished) of midline suboccipital craniectomies in which the dural substitute disintegrated or dissolved upon reexploration for pseudomeningocele repair. Although our experience with the fascial sling technique is limited, our preliminary results in 8 patients suggest that it is a safe and effective method for dural reconstruction of presigmoid dural defects following translabyrinthine resection of acoustic neuromas. Furthermore, additional postoperative lumbar drainage to divert CSF pressure away from the repair site does not appear to be necessary. Therefore, patients can avoid potential risks related to lumbar drainage including intracranial hypotension, CSF infection from an indwelling catheter, and thromboembolic complications from lack of mobilization. Patients who do not require lumbar drainage tend to ambulate sooner with a faster recovery and shorter hospital stays.

Successful prevention of CSF leak after translabyrinthine removal of acoustic neuromas depends significantly on careful and meticulous attention to surgical technique at every step of the closure. These steps include suturing the dural sling, sealing off the aditus ad antrum and mastoid air cells, applying tissue sealant for reinforcement, fat packing of the mastoid cavity, performing a cranioplasty of the mastoid defect to apply pressure on the fat graft, and precise multilayered wound closure. ${ }^{11}$

\section{Conclusions}

The dural sling reconstruction technique using autologous fascia can be safely and successfully used to reconstruct presigmoid dural defects after translabyrinthine resection of acoustic neuromas. Additional studies in a larger cohort of patients are warranted to ascertain whether this technique results in significantly lower rates of postoperative CSF leakage than other traditional dural reconstruction techniques.

\section{Disclosure}

The authors report no conflict of interest concerning the materials or methods used in this study or the findings specified in this paper.

Author contributions to the study and manuscript preparation include the following. Conception and design: Liu. Acquisition of data: all authors. Analysis and interpretation of data: Liu, Patel, Podolski. Drafting the article: Liu, Podolski, Patel. Critically revising the article: Liu, Patel. Reviewed submitted version of manuscript: Liu, Patel, Jyung. Approved the final version of the manuscript on behalf of all authors: Liu, Patel. Administrative/technical/ material support: Patel. Study supervision: Liu.

\section{References}

1. Arriaga MAC, Chen DA, Burke EL: Hydroxyapatite cement 
Fascial sling dural reconstruction for translabyrinthine approach

cranioplasty in translabyrinthine acoustic neuroma surgeryupdate. Otol Neurotol 28:538-540, 2007

2. Bambakidis NC, Munyon C, Ko A, Selman WR, Megerian CA: A novel method of translabyrinthine cranioplasty using hydroxyapatite cement and titanium mesh: a technical report. Skull Base 20:157-161, 2010

3. Becker SS, Jackler RK, Pitts LH: Cerebrospinal fluid leak after acoustic neuroma surgery: a comparison of the translabyrinthine, middle fossa, and retrosigmoid approaches. Otol Neurotol 24:107-112, 2003

4. Brackmann DE, Green JD Jr: Translabyrinthine approach for acoustic tumor removal. 1992. Neurosurg Clin N Am 19:251264, vi, 2008

5. Brennan JW, Rowed DW, Nedzelski JM, Chen JM: Cerebrospinal fluid leak after acoustic neuroma surgery: influence of tumor size and surgical approach on incidence and response to treatment. J Neurosurg 94:217-223, 2001

6. Celikkanat SM, Saleh E, Khashaba A, Taibah A, Russo A, Mazzoni A, et al: Cerebrospinal fluid leak after translabyrinthine acoustic neuroma surgery. Otolaryngol Head Neck Surg 112:654-658, 1995

7. Falcioni M, Mulder JJ, Taibah A, De Donato G, Sanna M: No cerebrospinal fluid leaks in translabyrinthine vestibular schwannoma removal: reappraisal of 200 consecutive patients. Am J Otol 20:660-666, 1999

8. Fayad JN, Schwartz MS, Slattery WH, Brackmann DE: Prevention and treatment of cerebrospinal fluid leak after translabyrinthine acoustic tumor removal. Otol Neurotol 28:387390, 2007

9. Fishman AJ, Hoffman RA, Roland JT Jr, Lebowitz RA, Cohen NL: Cerebrospinal fluid drainage in the management of CSF leak following acoustic neuroma surgery. Laryngoscope 106:1002-1004, 1996

10. Fishman AJ, Marrinan MS, Golfinos JG, Cohen NL, Roland JT Jr: Prevention and management of cerebrospinal fluid leak following vestibular schwannoma surgery. Laryngoscope 114:501-505, 2004

11. Goddard JC, Oliver ER, Lambert PR: Prevention of cerebrospinal fluid leak after translabyrinthine resection of vestibular schwannoma. Otol Neurotol 3:473-477, 2010

12. Hoffman RA: Cerebrospinal fluid leak following acoustic neuroma removal. Laryngoscope 104:40-58, 1994

13. House WF, Hitselberger WE: Transtemporal bone microsurgical removal of acoustic neuromas. Total versus subtotal removal of acoustic tumors. Arch Otolaryngol 80:751-752, 1964

14. Lanman TH, Brackmann DE, Hitselberger WE, Subin B: Report of 190 consecutive cases of large acoustic tumors (vestibular schwannoma) removed via the translabyrinthine approach. J Neurosurg 90:617-623, 1999

15. Liu JK, Saedi T, Delashaw JB Jr, McMenomey SO: Management of complications in neurotology. Otolaryngol Clin North Am 40:651-667, x-xi, 2007

16. Mamikoglu B, Wiet RJ, Esquivel CR: Translabyrinthine approach for the management of large and giant vestibular schwannomas. Otol Neurotol 23:224-227, 2002

17. Mangus BD, Rivas A, Yoo MJ, Alvarez J, Wanna GB, Haynes
DS, et al: Management of cerebrospinal fluid leaks after vestibular schwannoma surgery. Otol Neurotol 32:1525-1529, 2011

18. Mass SC, Wiet RJ, Dinces E: Complications of the translabyrinthine approach for the removal of acoustic neuromas. Arch Otolaryngol Head Neck Surg 125:801-804, 1999

19. Merkus P, Taibah A, Sequino G, Sanna M: Less than $1 \%$ cerebrospinal fluid leakage in 1,803 translabyrinthine vestibular schwannoma surgery cases. Otol Neurotol 31:276-283, 2010

20. Nutik SL, Korol HW: Cerebrospinal fluid leak after acoustic neuroma surgery. Surg Neurol 43:553-557, 1995

21. Panse R: Klinische und pathologische Mitteilungen IV. Ein Gliom des Akustikus. Arch Ohrenhielkd 61:215-255, 1904

22. Raslan AM, Liu JK, McMenomey SO, Delashaw JB Jr: Staged resection of large vestibular schwannomas. Clinical article. J Neurosurg 116:1126-1133, 2012

23. Ray J, D’Souza AR, Chavda SV, Walsh AR, Irving RM: Dissemination of fat in CSF: a common finding following translabyrinthine acoustic neuroma surgery. Clin Otolaryngol 30: 405-408, 2005

24. Reece AT, O'Reilly B, Teasdale E, Todd NV: Subarachnoid fat embolism complicating autologous fat grafting following translabyrinthine excision of acoustic neuroma. J Laryngol Otol 103:870-871, 1989

25. Ricaurte JC, Murali R, Mandell W: Uncomplicated postoperative lipoid meningitis secondary to autologous fat graft necrosis. Clin Infect Dis 30:613-615, 2000

26. Sanna M, Falcioni M, Rohit: Cerebro-spinal fluid leak after acoustic neuroma surgery. Otol Neurotol 24:524, 2003 (Letter)

27. Selesnick SH, Liu JC, Jen A, Newman J: The incidence of cerebrospinal fluid leak after vestibular schwannoma surgery. Otol Neurotol 25:387-393, 2004

28. Taha AN, Almefty R, Pravdenkova S, Al-Mefty O: Sequelae of autologous fat graft used for reconstruction in skull base surgery. World Neurosurg 75:692-695, 2011

29. Tos M, Thomsen J, Harmsen A: Results of translabyrinthine removal of 300 acoustic neuromas related to tumour size. Acta Otolaryngol Suppl 452:38-51, 1988

30. Valtonen HJ, Poe DS, Heilman CB, Tarlov EC: Endoscopically assisted prevention of cerebrospinal fluid leak in suboccipital acoustic neuroma surgery. Am J Otol 18:381-385, 1997

31. Yuen HW, Chen JM: Reconstructive options for skull defects following translabyrinthine surgery for vestibular schwannomas. Curr Opin Otolaryngol Head Neck Surg 16:318-324, 2008

Manuscript submitted May 14, 2012.

Accepted June 4, 2012.

Please include this information when citing this paper: DOI: 10.3171/2012.6.FOCUS12168.

Address correspondence to: James K. Liu, M.D., 90 Bergen Street Suite 8100, Department of Neurological Surgery, New Jersey Medical School, University of Medicine and Dentistry of New Jersey, Newark, New Jersey 07101. email: james.liu@umdnj.edu. 